\title{
Time Intervals of the Electron Transitions between the Energy States in the Hydrogen Atom Calculated in a Non-Probabilistic Way
}

\author{
Stanisław Olszewski \\ Institute of Physical Chemistry, Polish Academy of Sciences, Warsaw, Poland \\ Email: olsz@ichf.edu.pl
}

How to cite this paper: Olszewski, S. (2019) Time Intervals of the Electron Transitions between the Energy States in the Hydrogen Atom Calculated in a Non-Probabilistic Way. Journal of Modern Physics, 10, 1522-1531.

https://doi.org/10.4236/jmp.2019.1013101

Received: October 15, 2019

Accepted: November 12, 2019

Published: November 15, 2019

Copyright $\odot 2019$ by author(s) and Scientific Research Publishing Inc. This work is licensed under the Creative Commons Attribution International License (CC BY 4.0).

http://creativecommons.org/licenses/by/4.0/

\begin{abstract}
Definitions of the mechanical parameters entering the Bohr model of the hydrogen atom allowed us to calculate the time intervals connected with the electron transitions between the nearest-neighbouring energy levels in the atom. This is done in a strictly non-probabilistic way. The time results are compared with those derived earlier on the basis of the classical Joule-Lenz law for the energy emission adapted to the case of the electron transfer in the quantum systems. A similar formalism has been next applied to the harmonic oscillator and a particle moving in the one-dimensional potential box.
\end{abstract}

\section{Keywords}

Spectrum of the Hydrogen Atom, Time Intervals of the Electron Transitions

\section{Introduction}

Following the fundamental paper by Einstein [1] [2], a probabilistic approach to the intensity of the electron transitions in the atomic systems has been mainly applied: this has been done equally in the old as well as the modern quantum theory [2] [3] [4]. Physically this situation seems to be rather an encumbering one because the time intervals which are neglected are, in general, measurable parameters the size of which seems to be of interest for different processes.

A step towards a non-probabilistic theory of the electron transitions in the quantum systems has been done by the author [5] [6] [7]: it concerns the energy emission, therefore a transfer from a higher to a lower energy level, based on the classical Joule-Lenz law adapted to the quantum systems. When the transfer concerns solely the nearest-neighbouring quantum levels, say $n+1$ and $n$, the 
formula coupling the emitted energy $\Delta E$ to the emission time $\Delta t$ becomes very simple, namely

$$
\Delta E \Delta t=h
$$

here

$$
\Delta E=E_{n+1}-E_{n}>0 .
$$

Because of (1) and (2), the intensity of the energy emission from state $n+1$ to $n$ is represented by the ratio

$$
\frac{\Delta E}{\Delta t}
$$

The formula (3) could be next applied to the energy emission between the quantum states being also not nearest-neighbour ones. The numerical calculations were essentially limited to the ratios of the emission intensities for different kinds of the electron transitions in the hydrogen atom. The obtained results do not differ extensively from those calculated on the basis of the quantum-mechanical formalism; see [8] [9] [10].

Evidently (1) can be transformed into the formula representing $\Delta t$ :

$$
\Delta t=\frac{h}{\Delta E} \text {. }
$$

In case of large $n$ we have for the hydrogen atom

$$
\Delta E=-\frac{m e^{4}}{2 \hbar^{2}}\left[\frac{1}{(n+1)^{2}}-\frac{1}{n^{2}}\right] \cong \frac{m e^{4}}{2 \hbar^{2}} \cdot \frac{2 n+1}{n^{4}} \approx \frac{m e^{4}}{\hbar^{2} n^{3}},
$$

so in this case

$$
\Delta t=\frac{h}{\Delta E} \cong \frac{h \hbar^{2} n^{3}}{m e^{4}}=\frac{2 \pi \hbar^{3} n^{3}}{m e^{4}} .
$$

The aim of the present paper is to demonstrate that results for $\Delta t$ much similar to (6) can be easily obtained also by a direct application of the mechanical parameters entering the Bohr atomic model [11].

In Appendix A and Appendix B, respectively, the same formalism is applied to the harmonic linear oscillator and a particle enclosed in the one-dimensional potential box.

\section{The Time Interval $\Delta t$ Deduced from the Acceleration and Velocity Parameters Characteristic for the Bohr Atomic Model}

The main idea by Bohr in constructing his model of the hydrogen atom (see e.g. [11] [12]) was that the centrifugal acceleration force of the electron due to its circulation about the proton nucleus is compensated by the electrostatic attractive force acting between the proton and the electron. Therefore the mechanical acceleration is given by the formula

$$
a_{n}=\frac{v_{n}^{2}}{r_{n}}
$$


where

$$
v_{n}=\frac{e^{2}}{n \hbar}
$$

is the velocity size along the circular electron orbit (see e.g. [12]) and

$$
r_{n}=\frac{n^{2} \hbar^{2}}{m e^{2}}
$$

is the orbit radius [12]. Expressions (8) and (9) substituted into (7) give

$$
a_{n}=\frac{e^{4}}{n^{2} \hbar^{2}} \frac{m e^{2}}{n^{2} \hbar^{2}}=\frac{m e^{6}}{n^{4} \hbar^{4}} .
$$

In course of the electron transition from level $n+1$ to level $n$ the acceleration is changed by the interval whose absolute value is

$$
\begin{aligned}
\Delta a_{n} & =\left|a_{n+1}-a_{n}\right|=\left|\frac{m e^{6}}{\hbar^{4}}\left[\frac{1}{(n+1)^{4}}-\frac{1}{n^{4}}\right]\right| \\
& =\frac{m e^{6}}{\hbar^{4}} \frac{(n+1)^{4}-n^{4}}{(n+1)^{4} n^{4}} \approx \frac{m e^{6}}{\hbar^{4}} \frac{2 n 2 n^{2}}{n^{8}}=\frac{4 m e^{6}}{\hbar^{4} n^{5}},
\end{aligned}
$$

on condition in the last steps of (11) a large $n$ is only considered. Simultaneously the absolute change of the electron velocity becomes

$$
\Delta v_{n}=\left|\frac{e^{2}}{\hbar}\left(\frac{1}{n+1}-\frac{1}{n}\right)\right|=\frac{e^{2}}{\hbar} \frac{n+1-n}{(n+1) n} \approx \frac{e^{2}}{\hbar n^{2}},
$$

where the result obtained in the last step holds for large $n$. Since we expect the approximate validity of the formula

$$
\frac{\Delta v_{n}}{\Delta t}=\Delta a_{n},
$$

we obtain from (11)-(13) the time interval

$$
\Delta t=\frac{\Delta v_{n}}{\Delta a_{n}}=\frac{e^{2}}{n^{2} \hbar} \frac{\hbar^{4} n^{5}}{4 m e^{6}}=\frac{n^{3} \hbar^{3}}{4 m e^{4}} .
$$

The ratio of the result calculated in (6) to that obtained in (14) becomes equal to a constant number:

$$
\frac{2 \pi n^{3} \hbar^{3}}{m e^{4}} \cdot \frac{4 m e^{4}}{n^{3} \hbar^{3}}=8 \pi .
$$

\section{The Time Interval $\Delta t$ Deduced from the Electron Velocity and Orbit Length Parameters of the Bohr Model}

A still more simple calculation of $\Delta t$ than in Section 2 is connected with the case when the changes of the orbit length and electron velocity are taken into account. Here the change of velocity due to the electron transition between the levels $n+1$ and $n$ is given by the formula

$$
\frac{2 \pi \Delta r_{n}}{\Delta t}=\Delta v_{n}
$$


where

$$
\Delta r_{n}=r_{n+1}-r_{n}=\left[(n+1)^{2}-n^{2}\right] \frac{\hbar^{2}}{m e^{2}} \cong 2 n \frac{\hbar^{2}}{m e^{2}}
$$

and

$$
\Delta v_{n} \cong \frac{e^{2}}{\hbar} \frac{1}{n^{2}}
$$

is a repetition of the result obtained in (12). In effect from (16) we have

$$
\Delta t=\frac{2 \pi \Delta r_{n}}{\Delta v_{n}}=4 \pi n \frac{\hbar^{3}}{m e^{2}} \frac{\hbar n^{2}}{e^{2}}=\frac{4 \pi \hbar^{3} n^{3}}{m e^{4}} .
$$

This result for $\Delta t$ differs from that calculated in (6) solely by the factor of 2 .

\section{Time Interval of the Electron Transition and the Virial Theorem}

Let us examine here the time interval $\Delta t$ in reference to the virial theorem. Because of virial we have the following relation between the kinetic and potential electron energy in the hydrogen atom for any state $n$ :

$$
2 E_{n}^{\mathrm{kin}}+E_{n}^{\mathrm{pot}}=0 .
$$

In effect of (20) the electron energy becomes

$$
E_{n}=E_{n}^{\mathrm{kin}}+E_{n}^{\mathrm{pot}}=-E_{n}^{\mathrm{kin}}=-\frac{m}{2} v_{n}^{2}
$$

which implies

$$
\left|\frac{\Delta E_{n}}{\Delta t}\right| \cong \frac{m}{2} 2 v_{n} \frac{\Delta v_{n}}{\Delta t}=m v_{n} \Delta a_{n}=m \frac{e^{2}}{n \hbar} \frac{4 m e^{6}}{\hbar^{4} n^{5}}=\frac{4 m^{2} e^{8}}{\hbar^{5} n^{6}}
$$

due to the formulae (8) and (11). By substituting the result in (5) for $\Delta E_{n}$ into (22) we obtain

$$
\Delta t=\frac{\Delta E_{n}}{4 m^{2} e^{8}} \hbar^{5} n^{6}=\frac{m e^{4}}{\hbar^{2} n^{3}} \frac{\hbar^{5} n^{6}}{4 m^{2} e^{8}}=\frac{\hbar^{3} n^{3}}{4 m e^{4}}
$$

which is the interval $\Delta t$ identical to that given in (14).

\section{Discussion}

It looks that neither Einstein nor his followers were able to calculate a definite size of $\Delta t$, the time interval associated with the electron transitions in the atom, which, in the present considerations, is limited to the hydrogen case. In fact, the former authors were occupied mainly with the time changes of the transition probabilities than the time interval itself.

Our present idea concerning the time calculation was associated with an application of the classical definitions of the mechanical parameters (orbital position, electron velocity and acceleration) entering the Bohr model of the hydrogen atom. All these definitions make reference to the time interval $\Delta t$ of the electron transition. But $\Delta t$ could be calculated earlier on the basis of the clas- 
sical Joule-Lenz law ([13] and [5] [6] [7]) adapted to the electron transitions between the nearest-neighbouring energy levels of the atom; see the end of Section 1.

In fact the essence of the formulae attained for $\Delta t$ with the aid of the mechanical parameters in the Bohr atom and the expression for $\Delta t$ obtained from the Joule-Lenz law is the same. The results of both approaches differ solely by the constant number represented in (15) or the factor of 2 derived at the end of Section 3.

Evidently the present semiclassical calculations do not take into account the degeneracy of the energy levels in the atom with respect to the electron angular momentum, or electron spin. In consequence the selection rules for transitions connected with the changes of the angular momentum could not be obtained; see [14].

As the end step we present the result of the numerical calculations of $\Delta t$ in the hydrogen atom. By taking the formula (6) we obtain for large $n$ :

$$
\Delta t=\frac{2 \pi \hbar^{3}}{m e^{4}} n^{3} \cong 2 \pi \frac{\left(1.06 \times 10^{-27}\right)^{3}}{9.1 \times 10^{-28} \times\left(4.8 \times 10^{-10}\right)^{4}} n^{3} \text { sec } \cong 1.5 \times 10^{-16} n^{3} \text { sec } .
$$

In consequence the intensity of a single energy transition-considered also for large $n$-is

$$
\frac{\Delta E}{\Delta t} \cong \frac{3 \times 10^{5}}{n^{6}} \frac{\mathrm{erg}}{\mathrm{sec}}
$$

because from (5):

$$
\Delta E=\frac{m e^{4}}{\hbar^{2} n^{3}} \cong \frac{5 \times 10^{-11}}{n^{3}} \mathrm{erg} .
$$

Both cases represented in (24) and (25) consider the quantum transition

$$
n+1 \rightarrow n
$$

for $n \gg 1$.

The derivations of the time interval done-classically-in the paper indicate that the size of $\Delta t$ does not depend appreciably on the origin of $\Delta t$ specifically whether we consider the process of the energy emission $(n+1 \rightarrow n)$, or a reversed process of the absorption of energy $(n \rightarrow n+1)$.

A separate problem concerns the situation when the electron transition, say that of the energy emission between the levels $n+\alpha$ and $n$, viz.

$$
n+\alpha \rightarrow n
$$

has

$$
\alpha>1 \text {. }
$$

In this case Equations (1) and (6) joining the intervals $\Delta t$ and $\Delta E$ do not apply.

In order to overcome this difficulty the energy differences between the levels 


$$
\begin{aligned}
& n+1 \text { and } n, \\
& n+2 \text { and } n+1, \\
& n+3 \text { and } n+2, \\
& \vdots \\
& n+\alpha \text { and } n+\alpha-1
\end{aligned}
$$

should be separately considered. For any pair of states entering (29) we can apply the formulae (1) and (6) which give us a set of the time intervals representing transitions between the succesive pairs of the energy levels indicated in (29).

A sum of these time intervals listed respectively as

$$
\begin{gathered}
\Delta t_{1}, \\
\Delta t_{2}, \\
\Delta t_{3}, \\
\vdots \\
\Delta t_{\alpha}
\end{gathered}
$$

provides us with the time interval between the levels $n+\alpha$ and $n$ indicated in (27). Examples of such situations for the energy emission in the hydrogen atom are examined in [8] [9] [10].

\section{Conflicts of Interest}

The author declares no conflicts of interest regarding the publication of this paper.

\section{References}

[1] Einstein, A. (1917) Physikalische Zeitschrift, 18, 121.

[2] Van der Waerden, B.L. (1967) Sources of Quantum Mechanics. Dover, New York.

[3] Slater, J.C. (1960) Quantum Theory of the Atomic Structure. McGraw-Hill, New York.

[4] Schiff, L.I. (1968) Quantum Mechanics. 3rd Edition, McGraw-Hill, New York.

[5] Olszewski, S. (2015) Journal of Modern Physics, 6, 1277. https://doi.org/10.4236/jmp.2015.69133

Olszewski, S. (2016) Journal of Modern Physics, 7, 162.

[6] Olszewski, S. (2016) Quantum Matter, 5, 664. https://doi.org/10.1166/qm.2016.1360

[7] Olszewski, S. (2016) Reviews in Theoretical Science, 4, 1. https://doi.org/10.1166/rits.2016.1066

[8] Olszewski, S. (2016) Journal of Modern Physics, 7, 827. https://doi.org/10.4236/jmp.2016.78076

[9] Olszewski, S. (2016) Journal of Modern Physics, 7, 1004. https://doi.org/10.4236/jmp.2016.79091

Olszewski, S. (2016) Journal of Modern Physics, 7, 2314.

[10] Olszewski, S. (2017) Journal of Computational and Theoretical Nanoscience, 14, 4086. https://doi.org/10.1166/jctn.2017.6791

[11] Bohr, N. (1922) The Theory of Spectra and the Atomic Constitution. Cambridge University Press, Cambridge. 
[12] Sommerfeld, A. (1931) Atombau und Spektrallinien. Vol. 1, 5th Edition, Vieweg, Braunschweig.

[13] Lass, H. (1950) Vector and Tensor Analysis. McGraw-Hill, New York. https://doi.org/10.1119/1.1932684

[14] Condon, E.U. and Shortley, G.M. (1970) The Theory of Atomic Spectra. University Press, Cambridge.

[15] Sommerfeld, A. (1949) Mechanik, Akademische Verlagsgesellschaft, Leipzig.

[16] Eyring, H., Walter, J. and Kimball, G.E. (1957) Quantum Chemistry. Wiley, New York. 


\section{Appendix A. Transition Time between the Energy Levels $\boldsymbol{n}+$ 1 and $\boldsymbol{n}$ of the Harmonic Oscillaror}

First we note that the averages of the kinetic and potential parts of the energy $W$ of a linear harmonic oscillator, viz.

$$
W=\frac{m}{2} v^{2}+\frac{k}{2} x^{2}
$$

are equal; $m$ is the mass, $k$, the oscillator constant [15]. This result can be obtained by substituting

$$
x=A \cos \omega t
$$

where $A$ is the amplitude and $\omega$ is the circular frequency of the oscillator. In effect

$$
v=\frac{\mathrm{d} x}{\mathrm{~d} t}=-A \omega \sin \omega t
$$

gives in average

$$
\frac{m}{2} \overline{v^{2}}=\frac{m}{2} A^{2} \omega^{2} \overline{\sin ^{2} \omega t}=\frac{m}{4} A^{2} \omega^{2}
$$

whereas

$$
\frac{k}{2} \overline{x^{2}}=\frac{k}{2} A^{2} \overline{\cos ^{2} \omega t}=\frac{k}{4} A^{2} .
$$

The (A4) and (A5) are equal due to the relation

$$
\omega=\left(\frac{k}{m}\right)^{1 / 2} \text {. }
$$

Evidently the amplitude $A=A_{n}$ is different for any quantum state of energy

$$
W=W_{n} \cong n \hbar \omega
$$

which holds on condition $n$ is a large integer number.

By considering the averages in (A4) and (A5) we have

$$
\bar{E}_{\text {kin }}=\frac{m}{2} \overline{v_{n}^{2}}=\frac{m}{4} A_{n}^{2} \frac{k}{m}=A_{n}^{2} \frac{k}{4}=\frac{1}{2} W_{n} \cong \frac{1}{2} n \hbar \omega .
$$

In effect

$$
A_{n} \cong \frac{(2 n \hbar \omega)^{1 / 2}}{k}
$$

and

$$
\overline{v_{n}} \cong\left(\overline{v_{n}^{2}}\right)^{1 / 2} \approx\left(\frac{1}{2}\right)^{1 / 2} A_{n}\left(\frac{k}{m}\right)^{1 / 2}=\left(\frac{1}{2}\right)^{1 / 2} A_{n} \omega .
$$

The change of $\bar{v}$ due to the transition from state $n+1$ to $n$ leads to:

$$
\Delta \overline{v_{n}} \approx\left(\frac{1}{2}\right)^{1 / 2}\left(A_{n+1}-A_{n}\right) \omega .
$$

On the other side the change of the average velocity $\bar{v}$ can be considered as a direct effect of the change of the amplitude $A$ in course of sometime interval 
$\Delta t:$

$$
\Delta \overline{v_{n}} \cong \frac{\Delta A_{n}}{\Delta t}=\left(A_{n+1}-A_{n}\right) \frac{1}{\Delta t} .
$$

A comparison of (A11) with (A12) leads to relation

$$
\left(\frac{1}{2}\right)^{1 / 2}\left(A_{n+1}-A_{n}\right) \omega=\left(A_{n+1}-A_{n}\right) \frac{1}{\Delta t}
$$

which can be transformed into

$$
\Delta t=\frac{2^{1 / 2}}{\omega}
$$

On the other hand, according to the Joule-Lenz law, we have

$$
\Delta t=\frac{h}{\Delta E}=\frac{h}{\hbar(n+1-n) \omega}=\frac{2 \pi}{\omega} .
$$

A similarity between the $\Delta t$ obtained from the particle mechanics in (A14) and $\Delta t$ calculated from the Joule-Lenz law in (A15) becomes evident.

\section{Appendix B. Transition Time between the} Nearest-Neighbouring Energy Levels of a Particle Enclosed in a One-Dimensional Potential Box

The parameters characterizing a free particle of mass $m$ in a one-dimensional box having the length $L$ are given e.g. in [16]. According to the kinetic energy formula the particle in a quantum level $n$ has the velocity

$$
v_{n}=\left(\frac{2 E_{n}}{m}\right)^{1 / 2}=\frac{1}{2} \frac{n h}{m L}
$$

because the particle kinetic energy is

$$
E_{n}=\frac{n^{2} h^{2}}{8 m L^{2}}
$$

Evidently the velocity change between the levels $n+1$ and $n$ is

$$
\Delta v_{n}=\frac{1}{m^{1 / 2}}\left[\left(2 E_{n+1}\right)^{1 / 2}-\left(2 E_{n}\right)^{1 / 2}\right] \text {. }
$$

The energy change between the neighbouring levels is

$$
\Delta E=E_{n+1}-E_{n}=\frac{(n+1)^{2}-n^{2}}{8 m L^{2}} h^{2} \cong \frac{2 n h^{2}}{8 m L^{2}}=\frac{n h^{2}}{4 m L^{2}} ;
$$

in the last steps the condition $n \gg 1$ is considered.

The $\Delta E$ in (B4) can be coupled with the energy change entering (B3) by the relation

$$
\frac{\Delta E}{E_{n+1}^{1 / 2}-E_{n}^{1 / 2}}=\frac{E_{n+1}-E_{n}}{E_{n+1}^{1 / 2}-E_{n}^{1 / 2}}=E_{n+1}^{1 / 2}+E_{n}^{1 / 2} \approx 2 E_{n}^{1 / 2}
$$

where the last step holds for large $n$.

The acceleration change $\Delta a_{n}$ of the particle associated with the levels change 
from $n+1$ to $n$ is

$$
\Delta a_{n}=\frac{\Delta v_{n}}{\Delta t}
$$

where $\Delta t$ is an unknown time interval. This interval can be obtained when the balance of energy change due to the change $\Delta a_{n}$ is compared with the energy decrease (B4). This gives the relation

$$
\Delta a_{n} m 2 L=\frac{\Delta v_{n}}{\Delta t} m 2 L=\Delta E=E_{n+1}-E_{n}
$$

in which we assumed that the energy loss is produced in course of a full free-particle oscillation in the box along the path length

$$
L+L=2 L \text {. }
$$

By substituting in (B7) the necessary parameters we obtain the following relation for $\Delta t$ entering (B6):

$$
\begin{aligned}
\Delta t & =\frac{2 L m \Delta v_{n}}{\Delta E}=\frac{2 L m}{\Delta E} \frac{2^{1 / 2}}{m^{1 / 2}}\left(E_{n+1}^{1 / 2}-E_{n}^{1 / 2}\right)=\frac{2^{3 / 2} L m^{1 / 2}}{E_{n+1}^{1 / 2}+E_{n}^{1 / 2}} \\
& \approx \frac{2^{3 / 2} L m^{1 / 2}}{2 E_{n}^{1 / 2}}=2^{1 / 2} \frac{L^{2} m}{n h} 8^{1 / 2}=\frac{4 L^{2} m}{n h} .
\end{aligned}
$$

The end result of (B9) can be compared with $\Delta t$ obtained from the Joule-Lenz law [5] [6]:

$$
\Delta t=\frac{h}{\Delta E}=h \frac{4 m L^{2}}{n h^{2}}=\frac{4 L^{2} m}{n h} .
$$

In fact the (B9) and (B10) are the identical expressions.

It should be noted that identity concerns also the velocity in (B1) and that obtained on the basis of the distance (B8):

$$
v_{n}=\frac{2 L}{\Delta t}=2 L \frac{n h}{4 L^{2} m}=\frac{n h}{2 L m} .
$$

